Gerade in Deutschland sind noch Defizite zu überwinden

\section{Regional Governance als Motor einer nachhaltigen Entwicklung?}

\section{Der dynamische Wandel von Wirtschaft, Gesellschaft und Umwelt verändert die Kontextbedingungen für eine Steuerung gesellschaftlicher Entwicklung grundle- gend. Im Spannungsfeld zwischen Globalisierungs- und Regionalisierungsten- denzen erweisen sich herkömmliche, staatliche Regelungsmechanismen als immer weniger geeignet und in der Lage, die Herausforderungen zu bewältigen. Angesichts des Handlungsdrucks entwickeln sich auf der regionalen Ebene An- sätze never Steuerungsformen.}

I Von Guido Nischwitz und Reimar Molitor m Zusammenspiel mit dem Leitbild einer nachhaltigen Entwicklung ergeben sich im politischen Mehrebenensystem spürbare Verschiebungen, in denen die Steuerungskompetenzen und -fähigkeiten räumlicher, gesellschaftlicher und wirtschaftlicher Entwicklung neu gefasst werden. Dabei erfahren sowohl die globale als auch die regionale Handlungsebene eine deutliche Aufwertung. Gleichzeitig treten neue Steuerungsformen auf, die in der gesellschaftspolitischen Diskussion zunehmend mit dem Begriff Governance besetzt werden. Ähnlich wie bei Nachhaltigkeit besteht dabei eine hohe Anzahl unterschiedlichster Definitionen und Verwendungen. Governance wird in verschiedenen wirtschaftlichen, politischen und räumlichen Bezügen diskutiert: Corporate Governance, Good Governance, Sustainable Governance, Global Governance oder auch Local and Regional Governance.

In diesem Beitrag wird Governance als ein Instrument verstanden, das für eine Verknüpfung der drei zentralen Steuerungsformen Markt, Hierarchie und Netzwerke/Zivilgesellschaft steht. Governance umfasst somit jene institutionellen und gesellschaftlichen Strukturen, Politiken, Entscheidungsprozesse, formelle sowie informelle Regelungen, die sich auf die gesellschaftliche Auseinandersetzung mit den Themen Macht, Entscheidung und Partizipation konzentrieren (1). Im Gegensatz zum enger definierten staatlichen Steuerungssystem Government öffnet bzw. eröffnet Governance durch seine bewusste Unschärfe und Offenheit neue Möglichkeiten der Steuerung gesellschaftlicher Entwicklung, gerade auch für eine nachhaltige Entwicklung. Governance ist damit mehr als Government und als eine Verknüpfung von Akteuren in einem Netzwerk.
In vielen Handlungsfeldern, wie Verkehr und Mobilität, Landwirtschaft und Ernährung, Bauen und Wohnen oder Ver- und Entsorgung, erweist sich die Region zunehmend als zentrale Ebene für eine problemadäquate Abstimmung und Koordinierung. Konflikte und Problemlagen sowie entsprechende Lösungsmöglichkeiten sind für die Akteure vor Ort unmittelbarer zu erfahren und zu erarbeiten. Regional Governance bezeichnet den hierfür notwendigen Kommunikations- und Steuerungsprozess. Sie stellt eine Möglichkeit zur Komplexitätsreduktion gesellschaftlichen Wandels im Sinne einer Abbildung und Erklärung von Entwicklung im Raum dar. Die Anwendungsrelevanz ergibt sich aus der strategischen Bedienung der räumlichen Umsetzungsebene, die im Mittelpunkt der Alltags- und Arbeitswelt der Akteure steht. Regional Governance wird darüber hinaus häufig als Chance verstanden, eine nachhaltige Raumentwicklung zu initiieren, befördern und zu steuern (3). Zentrale Elemente sind:

- Neugestaltung der Interaktionen und Interdependenzen zwischen Administration, Wirtschaft und Zivilgesellschaft: Bildung und Erfassung neuer Akteurskonstellationen, Netzwerke und Kooperationen, Aufbau neuer und Wandel bestehender Institutionen und Instrumentarien.

- Etablierung von Kommunikationsprozessen als das konstituierende Element regionaler Entwicklungsprozesse.

- Entfaltung kontextbezogener räumlicher $\mathrm{Zu}$ schnitte, zum Beispiel thematische Regionen oder Regionen auf Zeit, jenseits administrativer Grenzen. In Deutschland steht man vor der besonderen Herausforderung, zwischen der stark hierarchisierten Raumordnung bzw. der Regionalpolitik sowie den projektbezogenen Regionszuschnitten in der Praxis zu vermitteln.

- Heterogenität regionaler Zielsetzungen und Steuerungsformen (Governance-Styles).

- Bestimmung gemeinsamer regionaler Entwicklungsleitbilder (,,common grounds“).

- Paradigmenwechsel von einer relativ statischen Planung von Entwicklung hin zu einem dynamischen Initiieren, Formen und Begleiten regionaler Entwicklung.

- Verlagerung von Entscheidungs- und Handlungskompetenzen auf die regionale Ebene; Stärkung regionaler Selbstorganisation.

\section{Erfahrungen in Deutschland}

Im Gegensatz zum europäischen Diskurs ist der räumliche Zugang zu Governance in Deutschland bislang nur sehr eingeschränkt aufgegriffen wor- 
den. Kennzeichnend ist eine eher positivistisch geprägte Auseinandersetzung mit Governance, die beim räumlichen Bezug häufig zu stark in sektoralen Zugängen und administrativen Konstrukten verhaftet bleibt. Zwischen dem Anspruch an eine ganzheitliche und integrative Sichtweise und den eher disziplinverhafteten Beiträgen klafft ein bedeutender Widerspruch. Eine Aufarbeitung dieser Defizite steht erst am Anfang. Ein Management der Schnittstellen zwischen den verschiedenen räumlichen, gesellschaftlichen und wirtschaftlichen Governance-Ebenen fehlt (4).

Bei der inhaltlichen Beschreibung von Regional Governance lassen sich anhand einer Expertenbefragung des IÖW, Wuppertal, in Deutschland grob drei Positionen unterscheiden:

- Netzwerkbildung / Akteursbezug: ein Zusammenwirken öffentlicher und privater Akteure zur Steuerung räumlicher Entwicklungsprozesse.

- Integrierte Zusammenführung zentraler Steuerungsformen: eine Kombination traditioneller harter und weicher Steuerungsansätze.

\section{- Aufbau von informellen Parallelstrukturen:} Bildung informeller Kooperationsformen parallel und teilweise auch in Konkurrenz zu etablierten politisch-administrativen Strukturen.

Regional Governance wird demnach noch eng in Abgrenzung zur hierarchisch verfassten Raumordnung bestimmt und auf informelle Akteursstrukturen bezogen. Diese Definition verstärkt den enormen Legitimationsdruck, dem das Konzept unterliegt. Die Fragen nach der demokratischen Legitimation, den Effektivitäts- und Legitimitätsgewinnen, Transaktionskosten, Grenzen und Gefahren sowie der Kontrolle bleiben bislang unbeantwortet.

Eng mit diesem Forschungsbedarf ist die Frage nach empirischen Beispielen für Regional Governance verbunden. Sind Lokale und Regionale Agenda 21Prozesse oder Public-private-Partnerships bereits Fallbeispiele für Governance? Angesichts des bislang nicht gelösten Problems der Einbeziehung von Wirtschaft und Unternehmen in Agenda-Prozesse oder der Nichtberücksichtigung neuer Akteurskonstellationen in Planungsvorgängen erscheint dies mehr als fraglich. Insofern überrascht es auch nicht, dass bei der Benennung entscheidender Akteure ein ,bunter Blumenstrauß“ bestehend aus jeweils relevanten Akteuren bzw. stakeboldern präsentiert wird, was wiederum die Frage nach einer generellen Übertragbarkeit aufwirft.

Regional Governance lässt derzeit in der deutschen Debatte viele Forschungsfragen offen:
Welche institutionellen und instrumentellen Voraussetzungen benötigt Regional Governance? - Welche regionsspezifischen Ausgangslagen bilden welche Governance-Styles aus?

- Wer muss und darf in welcher Konstellation für die Region strategisch denken?

- Wer macht Governance und wie ist Regional Governance organisiert?

- Wie werden Nachhaltigkeitsprinzipien in Prozesse von Regional Governance eingebettet?

Regional Governance, verstanden als strategischer Prozess für eine nachhaltige Raumentwicklung, steht vor der entscheidenden Herausforderung, wie die Spielregeln, insbesondere der Kommunikationsprozesse, bestimmt werden. Auf europäischer Ebene schickt sich die EU-Kommission an, entsprechend abgestimmte Rahmenbedingungen spätestens ab 2007 einzuführen. Regionen, die derzeit Governance-Strukturen vor Ort testen, haben sich - auch mit Blick auf die Fördermittelakquisition - auf den Weg in Richtung Zukunftsfähigkeit gemacht (5).

\section{Anmerkungen}

(1) United Nations Development Programme (UNDP

1997); im Internet unter http://magnet.undp.org

(2) Bundesamt für Bauwesen und Raumordnung (Hrsg.):

Handbuch Gute Beispiel einer nachhaltigen regionalen

Raum- und Siedlungsentwicklung, Studie des IÖW. BBR,

Werkstatt: Praxis Nr. 1/2000, www.zukunftsregionen.de

(3) European Commission: Sustainable governance. Institutional and procedural aspects of sustainability, Luxembourg 2000; European Commission: Consultation paper for the preparation of a European Union strategy for Sustainable Development, Luxembourg 2001.

(4) Im Rahmen des BMBF-Förderschwerpunkts Sozial-ökologische Forschung bearbeiteten die Autoren das Sondierungsprojekt "Local und Regional Governance für eine nachhaltige Entwicklung". Unter anderem wurden dabei auf internationaler und nationaler Ebene Experten befragt.

(5) Vgl. dazu auch die Aktivitäten des European Network on Sustainable Urban and Regional Development (ENSURE), www.european-association.org/ensure. Reimar Molitor ist für das IÖW im Executive Committee tätig.

\section{Die Autoren}

Dr. Guido Nischwitz leitet das Forschungsfeld Regionale Wirtschaftspolitik/Nachhaltige Regionalentwicklung im IÖW. Dr. Reimar Molitor ist seit Anfang dieses Jahres bei der Regionale 2006 Agentur im Bergischen Städtedreieck tätig.

Kontakt: IÖW-Regionalbüro NRW, Völklinger Str. 9, 42285 Wuppertal, Tel. 0202/ 80530, Fax 0202/

83402, E-mail: mailbox@wuppertal.ioew.de

\section{Neu im ökom verlag:}

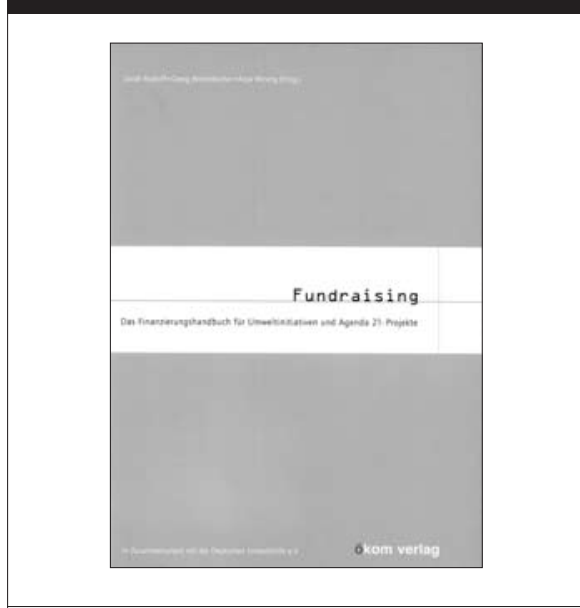

\section{Fundraising}

Das Finanzierungshandbuch für Umweltinitiativen und Agenda 21-Projekte

Geld- und Kooperationspartner fallen nicht vom Himmel. Das wissen auch die Umwelt- und Agenda 21-Initiativen, die sich ständig auf der Suche nach passenden Förderern und Partnern für Projekte befinden. Gerade kleine Initiativen können Berge versetzen, wenn gute Ideen, ein durchdachtes Konzept und die richtigen Menschen zusammentreffen.

Das neue Handbuch Fundraising zeigt, wie Finanzierungsprobleme erfolgreich gelöst werden können. Auf über 400 Seiten finden Projektinitiativen alles zum Thema Finanzierung. In dieser Form einzigartig im deutschsprachigen Raum sind die umfangreichen und übersichtlich dargestellten Porträts von rund 100 Umweltstiftungen.

Über 40 Fundraising-PraktikerInnen haben an diesem Handbuch mitgewirkt. Sie informieren über die aktuellen Fundraisingtrends sowie die neuen Möglichkeiten, die sich heute durch das Internet ergeben.

Mit Beiträgen von Marita Haibach, Gerhard Wallmeyer, Angela Scheibe-Jaeger, Hans-Josef Hönig, Christoph Müllerleile, Barbara Crole, Bettina Hohn, Rupert Graf Strachwitz, Pia Büber, Klaus-Dieter Boll u.v.a.

\section{www.oekom.de}

Herausgegeben von

Jacob Radloff, Georg R. Rettenbacher

und Anja Wirsing,

ökom Verlag, 416 Seiten, 48 DM,

ISBN 3-928244-59-0

Erhältlich bei pan adress, Semmelweisstraße 8

D - 82152 Planegg, Fon ++49/(0)89/85709-145,

Fax -131 oder unter www.oekom.de 
(c) 20I0 Authors; licensee IÖW and oekom verlag. This is an article distributed under the terms of the Creative Commons Attribution Non-Commercial No Derivates License (http://creativecommons.org/licenses/by-nc-nd/3.o/), which permits unrestricted use, distribution, and reproduction in any medium, provided the original work is properly cited. 